\title{
Diversity-oriented synthesis as a strategy for fragment evolution
}

\section{against GSK3ß}

Yikai Wang, Jean-Yves Wach, Patrick Sheehan, Cheng Zhong, Chenyang Zhan, Richard Harris,

Steven C. Almo, Joshua Bishop, Stephen J. Haggarty, Alexander Ramek, Kayla Berry, Conor

O'Herin, Angela N. Koehler, Alvin W. Hung*, and Damian W. Young*

Contents:

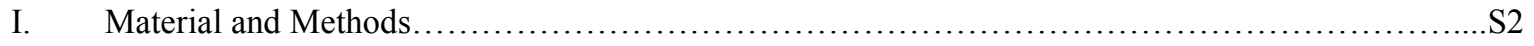

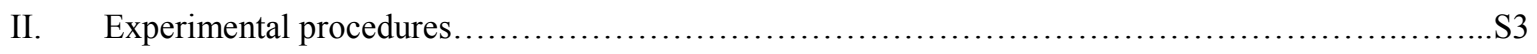

A. Chemical structures of synthetic fragments in the screening collection and PMI analysis......S3

B. General procedure for the synthesis of hit compounds and analogues....................S5

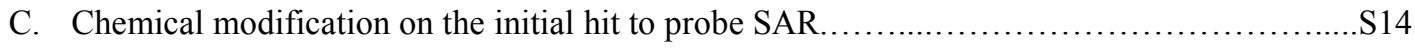

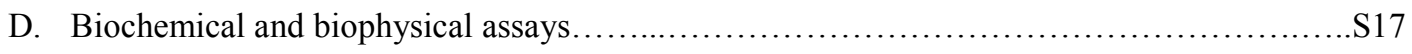

E. Crystallization, structure determination and refinement............................. 19

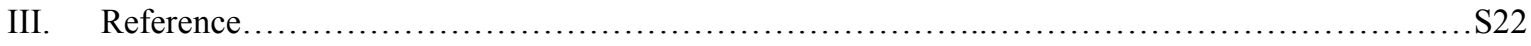




\section{Material and Methods}

Except as otherwise noted, reactions were carried out under argon. All reaction solvents except acetone were dispensed from a solvent purification system wherein solvents are passed through a packed activated alumina column. Acetone was Aldrich $99.5+\%$ histological grade. NMR spectra were recorded at $500 \mathrm{MHz}$ or $300 \mathrm{MHz}$ using a Varian I-500 or M-300 instrument. Chemical shifts for ${ }^{1} \mathrm{H}-\mathrm{NMR}$ spectra are reported in parts per million downfield from tetramethylsilane and were referenced to residual protonated solvents $\left(\mathrm{CHCl}_{3}: \mathrm{d} 7.26, \mathrm{CH}_{3} \mathrm{OH}\right.$ : 3.34, DMSO: 2.54). Chemical shifts for ${ }^{13} \mathrm{C}-\mathrm{NMR}$ spectra are reported in parts per million downfield from tetramethylsilane and referenced to protonated solvent $\left(\mathrm{CHCl}_{3}\right.$ : $\mathrm{d} 77.0, \mathrm{CH}_{3} \mathrm{OH}$ : 49.9, DMSO: 39.5). Data are represented as follows: chemical shift (multiplicity [bs = broad singlet, $\mathrm{s}=$ singlet, $\mathrm{d}=$ doublet, $\mathrm{t}=$ triplet, $\mathrm{q}=$ quartet, $\mathrm{m}=$ multiplet], coupling constants in Hertz, integration). High-resolution mass spectra were obtained through the Harvard University mass spectrometry facility. Optical rotations were obtained using digital polarimeter Autopol IV (Rudolph research Analytical) with a $1 \mathrm{~mL}$ cell and a $1 \mathrm{dm}$ path length. All reactions were magnetically stirred and monitored by thin-layer chromatography (TLC) using various eluents (ethyl acetate:(EtOAc):hexanes 1:5, methanol (MeOH):dichloromethane(DCM) 1:10) on Merck silica gel $60 \mathrm{~F} 254$ precoated plates $(0.25 \mathrm{~mm})$. Flash chromatography was performed with either EtOAc:hexanes or methanol:dichloromethane solvents using Merck silica gel 60 (230-400 mesh) or using a CombiFlash companion system (Teledyne ISCO, Inc.) with pre-packed FLASH silica gel columns (Teledyne ISCO, Inc.). SFC/MS chromatography was performed with a Berger analytic SFC (Waters ZQ Mass Spectrometer) using $\mathrm{CO}_{2}$ and isopropanol as the mobile phase and using a Chiralpak ${ }^{\circledR}$ AD-H column purchased from Chiral Technology Inc. (column length: 
4.6x250mm, particle size: 5um). Melting points were obtained with a Fisher Scientific 12-144 melting point apparatus.

\section{A. Chemical structures of synthetic fragments in the screening collection and PMI analysis}

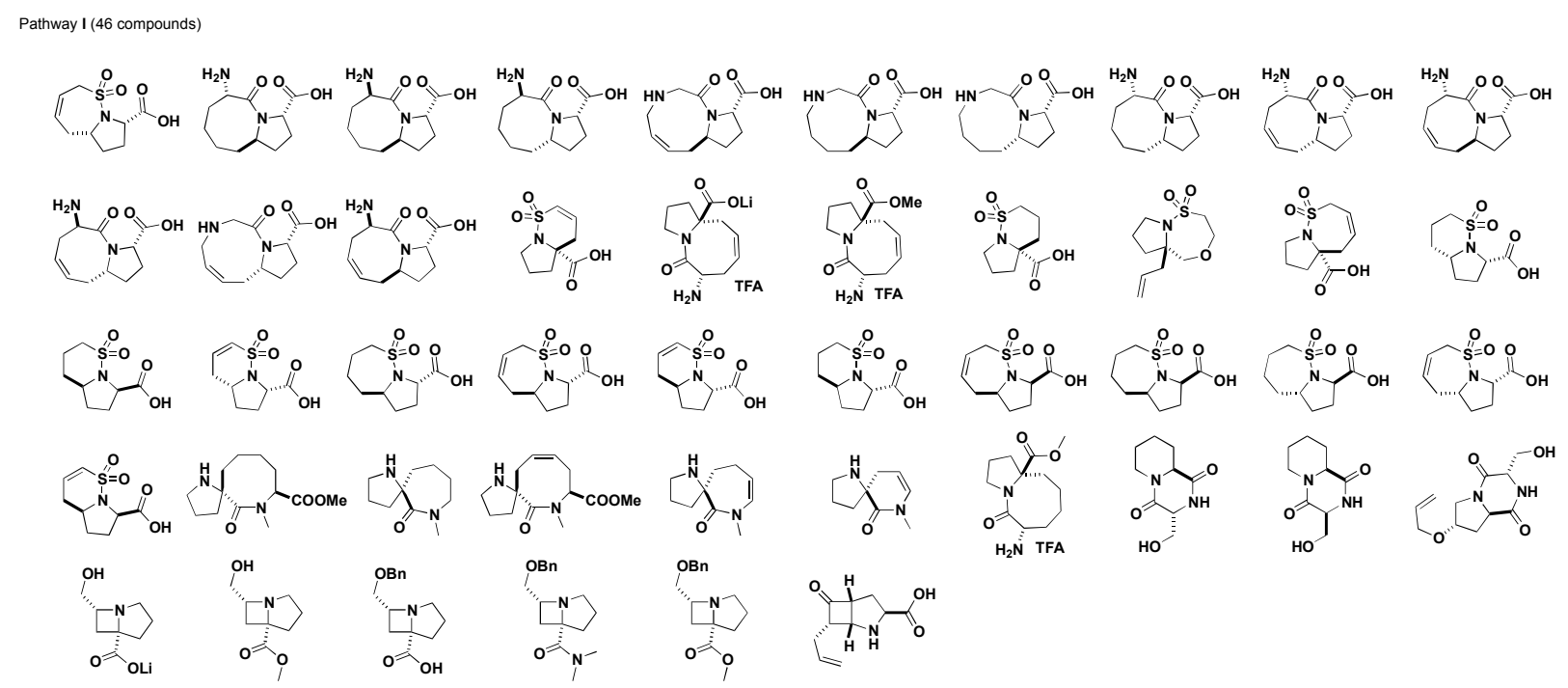

$$
\begin{aligned}
& \text { Pathway II (35 compounds) } \\
& \text { (racemic) } \\
& \text { (racemic) } \\
& \gamma_{H}^{O} \\
& \overbrace{H}^{O} \\
& \text { Pathway III (5 compounds) }
\end{aligned}
$$

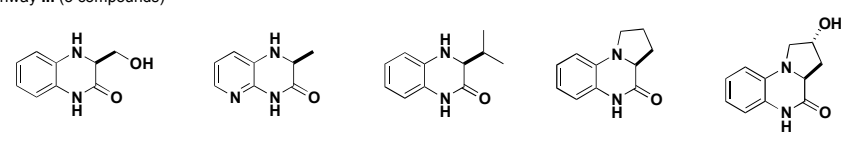

Figure S1. Fragment compounds in primary screening collection. 
For the synthesis and characterization of compounds from pathway I (Figure S1), see ref. 1. ${ }^{1}$ For synthesis and characterization of compounds from pathway II (Figure S1), see ref. 2. ${ }^{2}$ For synthesis and characterization of compounds from pathway III, see below.

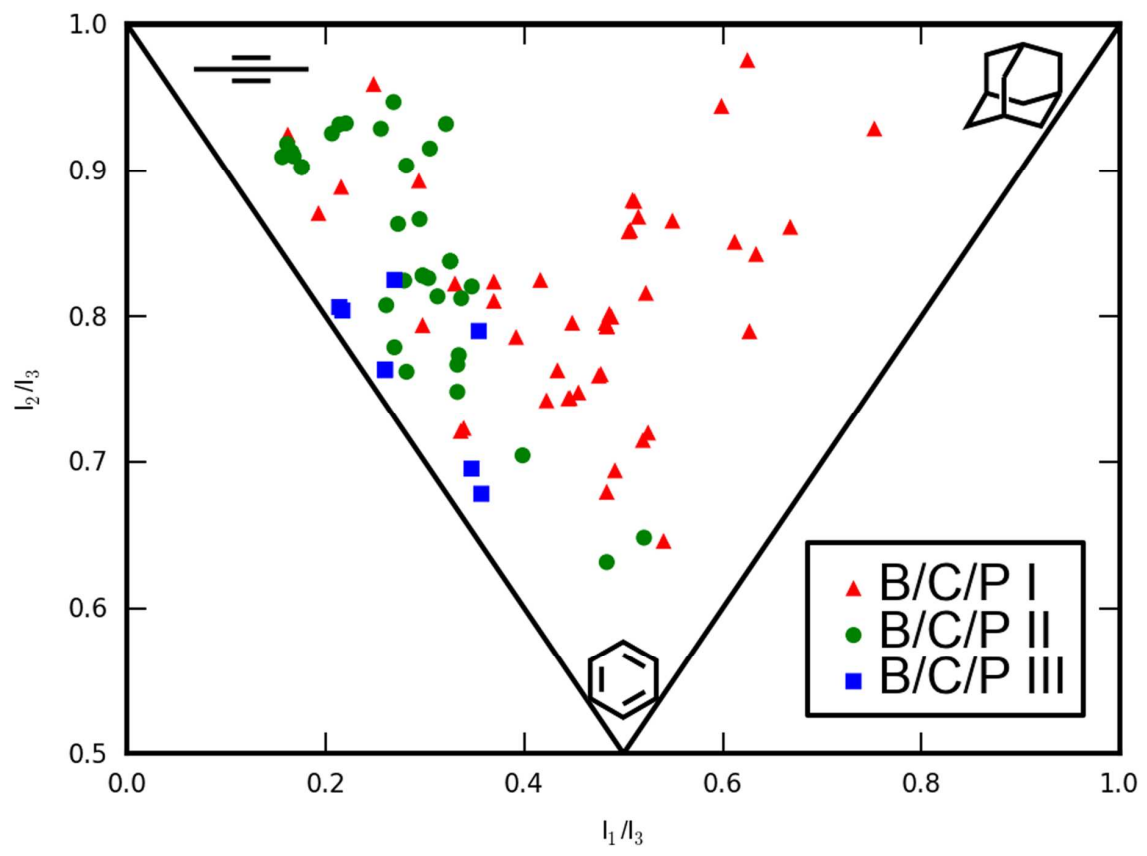

Figure S2. Principle moments of inertia (PMI) plot displaying shape diversity of the DOS fragments generated from the three pathways. ( $>86$ fragments were analyzed)

To access its shape diversity, the fragment library was analyzed computationally using a Principle Moments of Inertia plot $^{3,4}$ (Figure S2). Within each individual pathway, significant shape diversity is achieved which is a characteristic feature of DOS. Additionally, the composite shape diversity arising from the combination of the three pathways shows a wide distribution of molecular shapes in PMI space not encountered in traditional fragment collections. ${ }^{1}$ 


\section{B. General procedure for the synthesis of hit compounds and analogues}

Purities of all final compounds subjected to biological tests are larger than $95 \%$ based on LCMS or NMR analysis.

\section{General route for Pathway III}

General procedure for $\mathrm{S}_{\mathrm{N}} \mathrm{Ar}$ (adapted from ref. 5): ${ }^{5}$ to a stirred solution of 2-nitro-3fluoropyridine (1 equiv.) and methyl amino ester (or its hydrochloride salt, 2 equiv.) in DMSO $(0.5 \mathrm{M})$ was added DIPEA (4 equiv.) at room temperature. The resulting mixture was warmed up to $50{ }^{\circ} \mathrm{C}$ and stirred for 18 hours. The reaction was then cooled to room temperature, quenched with saturated $\mathrm{NaHCO}_{3}$ solution, extracted 3 times with EtOAc. The extract was washed with brine, dried with sodium sulfate, concentrated in vacuo and purified by column chromatography (EtOAc/hexane or $\mathrm{MeOH} / \mathrm{DCM}$ ) to afford the uncyclized product.

General procedure for cyclization: a solution of uncyclized nitro-containing compound (1 equiv.) and palladium on carbon (10 wt.\% Pd on carbon, wet, 0.05 equiv.) in solvent mixture of EtOAc and ethanol $(1: 1,0.1 \mathrm{M})$ was stirred at $25{ }^{\circ} \mathrm{C}$ under an atmosphere of hydrogen gas for 4 hours. The reaction flask was then purged with argon to replace hydrogen followed by the addition of ammonium formate ( 1 equiv.). The resulting mixture was warmed up to $40{ }^{\circ} \mathrm{C}$ and stirred for 18 hours. The suspension was then filtered through a Celite pad and the filtrate was concentrated in vacuo and purified by column chromatography (EtOAc/hexane or $\mathrm{MeOH} / \mathrm{DCM}$ ) to afford the cyclized product.

\section{Alternative route for Pathway III}

General procedure for Ullmann reaction/cyclodehydration sequence: adapted from the reported 
procedure, ${ }^{6}$ in a pressure tube, a suspension of 2-iodoaniline (1 equiv.), amino acid (2 equiv.), $\mathrm{K}_{3} \mathrm{PO}_{4}$ (2 equiv.), cuprous (I) chloride (2 mol\%), (E)-ethyl 2-cyano-2-(hydroxyimino)acetate (1 equiv.), and $N, N^{\prime}$-dimethylethylenediamine (DMEDA) (20 mol\%) in dry DMSO (0.3 M) was deoxygenated with argon and sealed with PTFE plug. The reaction mixture was then stirred at $120^{\circ} \mathrm{C}$ for $24 \mathrm{~h}$. The mixture was treated with water $(20 \mathrm{~mL})$ and the mixture was extracted three times with EtOAc. The combined organic layers were dried over anhydrous sodium sulfate. After filtration, solvent was evaporated to give the crude product, which was subjected to chromatography on silica gel (hexanes/EtOAc or DCM/MeOH) providing the desired compound.<smiles>CC1Nc2cccnc2NC1=O</smiles>

\section{(S)-2-methyl-1,2-dihydropyrido[2,3-b]pyrazin-3(4H)-one (1S)}

Yield 24\% over two steps, white powder; ${ }^{1} \mathrm{H}$ NMR (500 MHz, $\mathrm{d}_{6}$-DMSO): $\delta 10.7$ (s, $\left.1 \mathrm{H}\right), 7.61$ 7.59 (m, 1 H), 7.01-7.00 (m, 1 H), 6.84-6.81 (m, 1 H), 6.28 (s, 1 H), 3.92-3.88 (m, 1 H), 1.30 (d, $J=7.0 \mathrm{~Hz}, 3 \mathrm{H}) ;{ }^{13} \mathrm{C}$ NMR $\left(125 \mathrm{MHz}, \mathrm{d}_{6}\right.$-DMSO): $\delta 169.1,140.7,136.1,130.2,118.9,118.6$, 50.7, 17.7; mp: $212-215^{\circ} \mathrm{C} ;[\alpha]_{\mathrm{D}}^{23}=+102^{\circ}(\mathrm{c}=0.29$, in DMSO); HRMS (ESI-TOF): calculated for $\mathrm{C}_{8} \mathrm{H}_{10} \mathrm{~N}_{3} \mathrm{O}[\mathrm{M}+\mathrm{H}]^{+} 164.0818$, found 164.0821 .<smiles>C[C@H]1Nc2cccnc2NC1=O</smiles>

(R)-2-methyl-1,2-dihydropyrido[2,3-b]pyrazin-3(4H)-one (1R) 
Yield 46\% over two steps, light yellow powder; ${ }^{1} \mathrm{H}$ NMR (500 MHz, d6-DMSO): $\delta 10.7$ (s, $1 \mathrm{H}$ ), 7.61-7.59 (m, $1 \mathrm{H})$, 7.01-7.00 (m, $1 \mathrm{H}), 6.84-6.81(\mathrm{~m}, 1 \mathrm{H}), 6.28(\mathrm{~s}, 1 \mathrm{H}), 3.92-3.88(\mathrm{~m}, 1 \mathrm{H})$, $1.30(\mathrm{~d}, J=7.0 \mathrm{~Hz}, 3 \mathrm{H}) ;{ }^{13} \mathrm{C}$ NMR (125 MHz, $\mathrm{d}_{6}$-DMSO): $\delta 169.1,140.7,136.1,130.2,118.9$, 118.6, 50.7, 17.7; $\mathrm{mp} 217-220^{\circ} \mathrm{C} ;[\alpha]_{\mathrm{D}}^{23}=-98^{\circ}(\mathrm{c}=0.31$, in DMSO); HRMS (ESI-TOF): calculated for $\mathrm{C}_{8} \mathrm{H}_{10} \mathrm{~N}_{3} \mathrm{O}[\mathrm{M}+\mathrm{H}]^{+}$164.0818, found 164.0821.<smiles>CC1Nc2ccccc2NC1=O</smiles>

\section{(S)-3-methyl-3,4-dihydroquinoxalin-2(1H)-one (4S)}

Yield 50\% over two steps, white solid; ${ }^{1} \mathrm{H}$ NMR (500 MHz, $\mathrm{d}_{6}$-DMSO): $\delta 10.17$ (s, $\left.1 \mathrm{H}\right), 6.78$ $6.70(\mathrm{~m}, 4 \mathrm{H}), 6.68-6.66(\mathrm{~d}, J=10.0 \mathrm{~Hz}, 2 \mathrm{H}), 6.63-6.57$ (m, $2 \mathrm{H}), 6.0(\mathrm{bs}, 1 \mathrm{H}), 3.79-3.74$ (m, 1 H), $1.25(\mathrm{~d}, J=6.5 \mathrm{~Hz}, 3 \mathrm{H}) ;{ }^{13} \mathrm{C}-\mathrm{NMR}\left(125 \mathrm{MHz}, \mathrm{d}_{6}\right.$-DMSO): $\delta 169.0,135.3,126.8,123.3$, $118.4,115.3,113.9,51.6,18.1 ; \mathrm{mp} 117-119^{\circ} \mathrm{C} ;[\alpha]_{\mathrm{D}}^{23}=+125^{\circ}(\mathrm{c}=0.69$, in DMSO); HRMS (ESI-TOF): calculated for $\mathrm{C}_{9} \mathrm{H}_{11} \mathrm{~N}_{2} \mathrm{O}[\mathrm{M}+\mathrm{H}]^{+}$163.0866, found 163.0867 .<smiles>C[C@H]1Nc2ccccc2NC1=O</smiles>

\section{(R)-3-methyl-3,4-dihydroquinoxalin-2(1H)-one (4R)}

Yield 56\% over two steps, white solid; ${ }^{1} \mathrm{H}$ NMR (500 MHz, $\mathrm{d}_{6}$-DMSO): $\delta 10.17$ (s, $\left.1 \mathrm{H}\right), 6.80$ 6.70 (m, 4 H), 6.68-6.66 (m, 2 H), 6.63-6.57 (m, 2 H), 6.0 (bs, 1 H), 3.78-3.74 (m, 1 H), 1.25 (d, $J=6.5 \mathrm{~Hz}, 3 \mathrm{H}) ;{ }^{13} \mathrm{C}$ NMR (125 MHz, $\mathrm{d}_{6}$-DMSO) $\delta 168.4,134.3,126.3,122.3,117.7,114.6$, 
$113.2,50.5,17.4 ; \operatorname{mp~} 117-119{ }^{\circ} \mathrm{C} ;[\alpha]_{\mathrm{D}}^{23}=-130^{\circ}(\mathrm{c}=0.73$, in DMSO); HRMS (ESI-TOF): calculated for $\mathrm{C}_{9} \mathrm{H}_{11} \mathrm{~N}_{2} \mathrm{O}[\mathrm{M}+\mathrm{H}]^{+}$163.0866, found 163.0867.<smiles>CC1Nc2ccncc2NC1=O</smiles>

\section{(S)-2-methyl-1,2-dihydropyrido[3,4-b]pyrazin-3(4H)-one (5S)}

Yield $15 \%$ via the Ullmann reaction/cyclodehydration route, brown solid; ${ }^{1} \mathrm{H}$ NMR $(500 \mathrm{MHz}$, $\mathrm{d}_{6}$-DMSO): $\delta 7.79(\mathrm{~d}, J=5.5 \mathrm{~Hz}, 1 \mathrm{H}), 7.75(\mathrm{~s}, 1 \mathrm{H}), 6.63(\mathrm{~d}, J=5.5 \mathrm{~Hz}, 1 \mathrm{H}), 4.59(\mathrm{~s}, 2 \mathrm{H})$ 4.10 (q, $J=7.0 \mathrm{~Hz}, 1 \mathrm{H}), 1.40(\mathrm{~d}, J=7.0 \mathrm{~Hz}, 3 \mathrm{H}) ;{ }^{13} \mathrm{C}-\mathrm{NMR}\left(125 \mathrm{MHz}, \mathrm{d}_{6}\right.$-DMSO): $\delta 167.2$, $144.1,139.8,134.6,122.6,107.4,50.3,18.5 ; \mathrm{mp} 255-258{ }^{\circ} \mathrm{C} ;[\alpha]_{\mathrm{D}}^{23}=+15^{\circ}(\mathrm{c}=0.69$, in DMSO); HRMS (ESI-TOF): calculated for $\mathrm{C}_{8} \mathrm{H}_{10} \mathrm{~N}_{3} \mathrm{O}[\mathrm{M}+\mathrm{H}]^{+}$164.0818, found 164.0823.<smiles>C[C@H]1Nc2ccncc2NC1=O</smiles>

\section{(R)-2-methyl-1,2-dihydropyrido[3,4-b]pyrazin-3(4H)-one (5R)}

Yield 6\% via the Ullmann reaction/cyclodehydration route, brown solid; ${ }^{1} \mathrm{H}-\mathrm{NMR}\left(500 \mathrm{MHz}, \mathrm{d}_{6^{-}}\right.$ DMSO): $\delta 7.79$ (d, $J=5.5 \mathrm{~Hz}, 1 \mathrm{H}), 7.75(\mathrm{~s}, 1 \mathrm{H}), 6.63$ (d, $J=5.5 \mathrm{~Hz}, 1 \mathrm{H}), 4.59$ (s, $2 \mathrm{H}), 4.10$ $(\mathrm{q}, J=7.0 \mathrm{~Hz}, 1 \mathrm{H}), 1.40(\mathrm{~d}, J=7.0 \mathrm{~Hz}, 3 \mathrm{H}) ; \mathrm{d}_{6}$-DMSO) $\delta 167.2,144.1,139.8,134.6,122.6$, 107.4, 50.3, 18.5; mp $258-260{ }^{\circ} \mathrm{C} ;[\alpha]_{\mathrm{D}}^{23}=-14^{\circ}(\mathrm{c}=0.48$, in DMSO); HRMS (ESI-TOF): calculated for $\mathrm{C}_{8} \mathrm{H}_{10} \mathrm{~N}_{3} \mathrm{O}[\mathrm{M}+\mathrm{H}]^{+}$164.0818, found 164.0823. 
<smiles>CC1Nc2cnccc2NC1=O</smiles>

\section{(S)-3-methyl-3,4-dihydropyrido[3,4-b]pyrazin-2(1H)-one (6S)}

Following the general route for pathway III, the cyclization was performed with palladium on carbon (10 wt.\% Pd on carbon, wet, 0.05 equiv.) in solvent mixture of EtOAc and ethanol (1:1, $0.1 \mathrm{M}$ ) under an atmosphere of hydrogen gas at $40{ }^{\circ} \mathrm{C}$ for 18 hours; yield $41 \%$ over two steps, light yellow solid; ${ }^{1} \mathrm{H}$ NMR (500 MHz, $\left.\mathrm{CD}_{3} \mathrm{OD}\right): \delta 7.88(\mathrm{~s}, 1 \mathrm{H}), 7.79(\mathrm{~d}, J=4.0 \mathrm{~Hz}, 1 \mathrm{H}), 6.76$ $(\mathrm{d}, J=5.0 \mathrm{~Hz}, 1 \mathrm{H}), 3.97(\mathrm{q}, J=6.5 \mathrm{~Hz}, 1 \mathrm{H}), 3.34(\mathrm{~s}, 1 \mathrm{H}), 1.38(\mathrm{~d}, J=6.0 \mathrm{~Hz}, 3 \mathrm{H}) ;{ }^{13} \mathrm{C} \mathrm{NMR}$ (125 MHz, $\left.\mathrm{CD}_{3} \mathrm{OD}\right): \delta 168.7,139.7,134.6,132.4,130.9,109.1,50.7,17.5 ; \mathrm{mp} 250-253{ }^{\circ} \mathrm{C}$; $[\alpha]_{\mathrm{D}}^{23}=+60^{\circ}\left(\mathrm{c}=0.91\right.$, in DMSO); HRMS (ESI-TOF): calculated for $\mathrm{C}_{8} \mathrm{H}_{10} \mathrm{~N}_{3} \mathrm{O}[\mathrm{M}+\mathrm{H}]^{+}$ 164.0818, found 164.0823 .<smiles>C[C@H]1Nc2ccncc2NC1=O</smiles>

\section{(R)-3-methyl-3,4-dihydropyrido[3,4-b]pyrazin-2(1H)-one (6R)}

Following the general route for pathway III, the cyclization was performed with palladium on carbon (10 wt.\% Pd on carbon, wet, 0.05 equiv.) in solvent mixture of EtOAc and ethanol (1:1, $0.1 \mathrm{M}$ ) under an atmosphere of hydrogen gas at $40{ }^{\circ} \mathrm{C}$ for 18 hours; yield $35 \%$ over two steps, yellowish solid; ${ }^{1} \mathrm{H}$ NMR (500 MHz, $\left.\mathrm{CD}_{3} \mathrm{OD}\right): \delta 7.88$ (s, $\left.1 \mathrm{H}\right), 7.79$ (d, J=4.0 Hz, $\left.1 \mathrm{H}\right), 6.76$ (d, $J=5.0 \mathrm{~Hz}, 1 \mathrm{H}), 3.97(\mathrm{q}, J=6.5 \mathrm{~Hz}, 1 \mathrm{H}), 3.34(\mathrm{~s}, 1 \mathrm{H}), 1.38(\mathrm{~d}, J=6.0 \mathrm{~Hz}, 3 \mathrm{H}) ;{ }^{13} \mathrm{C} \mathrm{NMR}$ (125 MHz, $\left.\mathrm{CD}_{3} \mathrm{OD}\right): \delta 168.7,139.7,134.6,132.4,130.9,109.1,50.7,17.5 ; \mathrm{mp} 252-255{ }^{\circ} \mathrm{C}$; 
$[\alpha]_{\mathrm{D}}^{23}=-53^{\circ}\left(\mathrm{c}=0.93\right.$, in DMSO); HRMS (ESI-TOF): calculated for $\mathrm{C}_{8} \mathrm{H}_{10} \mathrm{~N}_{3} \mathrm{O}[\mathrm{M}+\mathrm{H}]^{+}$ 164.0818, found 164.0825 .<smiles>CC1Nc2ncccc2NC1=O</smiles>

\section{(S)-3-methyl-3,4-dihydropyrido[2,3-b]pyrazin-2(1H)-one (7S)}

Yield 18\% over two steps, light brown solid; ${ }^{1} \mathrm{H}$ NMR (500 MHz, $\mathrm{d}_{6}$-DMSO): $\delta 10.4$ (s, $1 \mathrm{H}$ ), 7.66-7.65 (m, $1 \mathrm{H}), 6.97-6.96(\mathrm{~m}, 1 \mathrm{H}), 6.85$ (s, $1 \mathrm{H}), 6.62-6.59$ (m, $1 \mathrm{H}), 4.05-4.01$ (m, $1 \mathrm{H})$, $1.33(\mathrm{~d}, J=7.0 \mathrm{~Hz}, 3 \mathrm{H}) ;{ }^{13} \mathrm{C}$ NMR $\left(125 \mathrm{MHz}, \mathrm{d}_{6}-\mathrm{DMSO}\right): \delta 167.5,147.0,141.0,120.8,119.9$, 113.3, 50.8, 18.5; mp 261-264 ${ }^{\circ} \mathrm{C} ;[\alpha]_{\mathrm{D}}^{23}=+57^{\circ}(\mathrm{c}=0.42$, in DMSO). HRMS (ESI-TOF): calculated for $\mathrm{C}_{8} \mathrm{H}_{10} \mathrm{~N}_{3} \mathrm{O}[\mathrm{M}+\mathrm{H}]^{+}$164.0818, found 164.0827 .<smiles>C[C@H]1Nc2ncccc2NC1=O</smiles>

\section{(R)-3-methyl-3,4-dihydropyrido[2,3-b]pyrazin-2(1H)-one (7R)}

Yield 33\% over two steps, light brown solid; ${ }^{1} \mathrm{H}$ NMR (500 MHz, $\mathrm{d}_{6}$-DMSO): $\delta 10.4$ (s, $1 \mathrm{H}$ ), 7.66-7.65 (m, $1 \mathrm{H}), 6.97-6.96(\mathrm{~m}, 1 \mathrm{H}), 6.85(\mathrm{~s}, 1 \mathrm{H}), 6.62-6.59(\mathrm{~m}, 1 \mathrm{H}), 4.05-4.01(\mathrm{~m}, 1 \mathrm{H})$, $1.33(\mathrm{~d}, J=7.0 \mathrm{~Hz}, 3 \mathrm{H}) ;{ }^{13} \mathrm{C}$ NMR (125 MHz, $\left.\mathrm{d}_{6}-\mathrm{DMSO}\right): \delta 167.5,147.0,141.0,120.8,119.9$, 113.3, 50.8, 18.5; mp 252-255 ${ }^{\circ} \mathrm{C} ;[\alpha]_{\mathrm{D}}^{23}=-41^{\circ}(\mathrm{c}=0.41$, in DMSO); HRMS (ESI-TOF): calculated for $\mathrm{C}_{8} \mathrm{H}_{10} \mathrm{~N}_{3} \mathrm{O}[\mathrm{M}+\mathrm{H}]^{+}$164.0818, found 164.0825. 
<smiles>CC(C)C1Nc2cccnc2NC1=O</smiles>

\section{(S)-2-isopropyl-1,2-dihydropyrido[2,3-b]pyrazin-3(4H)-one (12S)}

Yield 54\% over two steps, yellowish solid; ${ }^{1} \mathrm{H}$ NMR (500 MHz, $\left.\mathrm{d}_{6}-\mathrm{DMSO}\right): \delta 10.6$ (s, $1 \mathrm{H}$ ), 7.48-7.47 (m, $1 \mathrm{H}), 7.00-6.99(\mathrm{~d}, J=5 \mathrm{~Hz}, 1 \mathrm{H}), 6.76-6.73(\mathrm{~m}, 1 \mathrm{H}), 6.22(\mathrm{~s}, 1 \mathrm{H})$, 3.67-3.65 (m, $1 \mathrm{H}), 2.06-1.98(\mathrm{~m},, 1 \mathrm{H}), 0.94-0.93(\mathrm{~d}, J=5 \mathrm{~Hz}, 3 \mathrm{H}), 0.84-0.82(\mathrm{~d}, J=7 \mathrm{~Hz}, 3 \mathrm{H}) ;{ }^{13} \mathrm{C} \mathrm{NMR}$ (125 MHz, d6-DMSO); $\delta 168.3,140.4,135.9,130.8,119.4,118.7,61.4,32.1,19.4,18.1 ; \mathrm{mp}$ 162-165 ${ }^{\circ} \mathrm{C} ;[\alpha]_{\mathrm{D}}^{23}=+16^{\circ}\left(\mathrm{c}=1.3\right.$, in DMSO); HRMS (ESI-TOF) calculated for $\mathrm{C}_{10} \mathrm{H}_{14} \mathrm{~N}_{3} \mathrm{O}$ $[\mathrm{M}+\mathrm{H}]^{+}$192.1137, found 192.1139.<smiles>CC(C)[C@H]1Nc2cccnc2NC1=O</smiles>

\section{(R)-2-isopropyl-1,2-dihydropyrido[2,3-b]pyrazin-3(4H)-one (12R)}

Yield 45\% over two steps, light yellow solid; ${ }^{1} \mathrm{H}$ NMR (500 MHz, $\mathrm{d}_{6}$-DMSO): $\delta 10.62$ (s, $1 \mathrm{H}$ ), 7.48-7.47 (d, $J=5 \mathrm{~Hz}, 1 \mathrm{H}), 7.01-6.99$ (d, $J=10 \mathrm{~Hz}, 1 \mathrm{H}), 6.76-6.73$ (m, $1 \mathrm{H}), 6.22(\mathrm{~s}, 1 \mathrm{H})$, 3.67-3.66 (dd, $J=2.5 \mathrm{~Hz}, J=2.5 \mathrm{~Hz}, 1 \mathrm{H}), 2.02-2.01(\mathrm{~m}, 1 \mathrm{H}), 0.94-0.92(\mathrm{~d}, J=7 \mathrm{~Hz}, 3 \mathrm{H})$, 0.84-0.82 (d, $J=7 \mathrm{~Hz}, 3 \mathrm{H}) ;{ }^{13} \mathrm{C}$ NMR (125 MHz, d 6 -DMSO): $\delta 168.3,140.4,135.9,130.8$, $119.4,118.7,61.4,32.1,19.4,18.1 ; \mathrm{mp} 156-159^{\circ} \mathrm{C} ;[\alpha]_{\mathrm{D}}^{23}=-12^{\circ}(\mathrm{c}=1.3$, in DMSO $)$, HRMS (ESI-TOF): calculated for $\mathrm{C}_{10} \mathrm{H}_{14} \mathrm{~N}_{3} \mathrm{O}[\mathrm{M}+\mathrm{H}]^{+}$192.1137, found 192.1139. 
<smiles>O=C1Nc2ncccc2NC1Cc1ccccc1</smiles>

\section{(S)-2-benzyl-1,2-dihydropyrido[2,3-b]pyrazin-3(4H)-one (13S)}

Yield 45\% over two steps, yellow oil; ${ }^{1} \mathrm{H}$ NMR $\left(500 \mathrm{MHz}, \mathrm{CDCl}_{3}\right): \delta 10.67(\mathrm{~s}, 1 \mathrm{H}), 7.49(\mathrm{~d}, J=$ $5 \mathrm{~Hz}, 1 \mathrm{H}), 7.31-7.16(\mathrm{~m}, 3 \mathrm{H}), 6.97(\mathrm{~d}, J=10 \mathrm{~Hz}, 1 \mathrm{H}), 6.77(\mathrm{t}, J=15 \mathrm{~Hz}, 1 \mathrm{H}), 6.16(\mathrm{~s}, 1 \mathrm{H})$, 4.17-4.15 (m, $1 \mathrm{H}), 3.58(\mathrm{~s}, 1 \mathrm{H}), 3.36$ (bs, $1 \mathrm{H}), 2.99-2.89$ (m, 2H); ${ }^{13} \mathrm{C}-\mathrm{NMR}(125 \mathrm{MHz}$, $\left.\mathrm{CDCl}_{3}\right): \delta 167.5,139.9,137.0,135.7,129.8,129.5,128.0,126.3,118.8,118.7,56.6,38.2 ;[\alpha]_{\mathrm{D}}^{23}$ $=+13^{\circ}\left(\mathrm{c}=0.71\right.$, in DMSO); HRMS (ESI-TOF): calculated for $\mathrm{C}_{14} \mathrm{H}_{14} \mathrm{~N}_{3} \mathrm{O}[\mathrm{M}+\mathrm{H}]^{+} 240.1131$, found 240.1140 .<smiles>O=C1Nc2ncccc2N[C@H]1Cc1ccccc1</smiles>

\section{(R)-2-benzyl-1,2-dihydropyrido[2,3-b]pyrazin-3(4H)-one (13R)}

Yield 54\% over two steps, yellow oil; ${ }^{1} \mathrm{H}$ NMR $\left(500 \mathrm{MHz}, \mathrm{CDCl}_{3}\right): \delta 10.67(\mathrm{~s}, 1 \mathrm{H}), 7.49(\mathrm{~d}, J=$ $5 \mathrm{~Hz}, 1 \mathrm{H}), 7.31-7.16(\mathrm{~m}, 3 \mathrm{H}), 6.97(\mathrm{~d}, J=10 \mathrm{~Hz}, 1 \mathrm{H}), 6.77(\mathrm{t}, J=15 \mathrm{~Hz}, 1 \mathrm{H}), 6.16(\mathrm{~s}, 1 \mathrm{H})$, 4.17-4.15 (m, $1 \mathrm{H}), 3.58(\mathrm{~s}, 1 \mathrm{H}), 3.36(\mathrm{bs}, 1 \mathrm{H}), 2.99-2.89(\mathrm{~m}, 2 \mathrm{H}) ;{ }^{13} \mathrm{C} \mathrm{NMR}(125 \mathrm{MHz}$, $\left.\mathrm{CDCl}_{3}\right): \delta 167.5,139.9,137.0,135.7,129.8,129.5,128.0,126.3,118.8,118.7,56.6,38.2 ;[\alpha]_{\mathrm{D}}^{23}$ $=-25^{\circ}\left(\mathrm{c}=0.67\right.$, in DMSO); HRMS (ESI-TOF): calculated for $\mathrm{C}_{14} \mathrm{H}_{14} \mathrm{~N}_{3} \mathrm{O}[\mathrm{M}+\mathrm{H}]^{+} 240.1131$, found 240.1138 . 


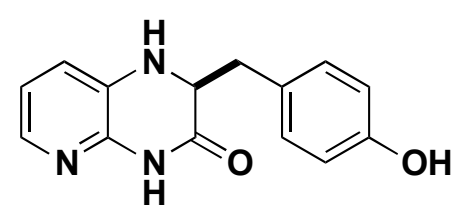

(S)-2-(4-hydroxybenzyl)-1,2-dihydropyrido[2,3-b]pyrazin-3(4H)-one (14S)

Yield 60\% over two steps, light yellow solid; ${ }^{1} \mathrm{H}$ NMR (500 MHz, $\mathrm{d}_{6}$-DMSO): $\delta 10.6$ (s, $1 \mathrm{H}$ ), $9.15(\mathrm{~s}, 1 \mathrm{H}), 7.47-7.46(\mathrm{~m}, 1 \mathrm{H}), 6.95-6.93(\mathrm{~m}, 3 \mathrm{H}), 6.74-6.72(\mathrm{~m}, 1 \mathrm{H}), 6.61(\mathrm{~d}, J=8.0 \mathrm{~Hz}, 2$ H), $6.04(\mathrm{~s}, 1 \mathrm{H}), 4.04-4.01(\mathrm{~m}, 1 \mathrm{H}), 2.82(\mathrm{dd}, J=13.5,4.5 \mathrm{~Hz}, 1 \mathrm{H}), 2.75(\mathrm{dd}, J=13.5,7.0 \mathrm{~Hz}$, $1 \mathrm{H}) ;{ }^{13} \mathrm{C}$ NMR (125 MHz, $\mathrm{d}_{6}$-DMSO): $\delta 167.8,155.8,139.9,135.5,130.6,129.5,126.8,118.7$, 118.6, 56.9, 37.4; mp $167-170{ }^{\circ} \mathrm{C} .[\alpha]_{\mathrm{D}}^{23}=+11^{\circ}(\mathrm{c}=0.59$, in DMSO); HRMS (ESI-TOF): calculated for $\mathrm{C}_{14} \mathrm{H}_{14} \mathrm{~N}_{3} \mathrm{O}_{2}[\mathrm{M}+\mathrm{H}]^{+}$256.1081, found 256.1087.

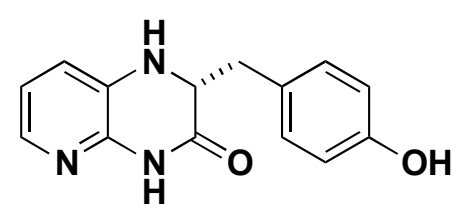

(R)-2-(4-hydroxybenzyl)-1,2-dihydropyrido[2,3-b]pyrazin-3(4H)-one (14R)

Yield 52\% over two steps, light yellow solid; ${ }^{1} \mathrm{H}$ NMR (500 MHz, $\mathrm{d}_{6}$-DMSO): $\delta 10.6$ (s, $1 \mathrm{H}$ ), $9.15(\mathrm{~s}, 1 \mathrm{H}), 7.47-7.46(\mathrm{~m}, 1 \mathrm{H}), 6.95-6.93(\mathrm{~m}, 3 \mathrm{H}), 6.74-6.72(\mathrm{~m}, 1 \mathrm{H}), 6.61(\mathrm{~d}, J=8.0 \mathrm{~Hz}, 2$ H), $6.04(\mathrm{~s}, 1 \mathrm{H}), 4.04-4.01(\mathrm{~m}, 1 \mathrm{H}), 2.82(\mathrm{dd}, J=13.5,4.5 \mathrm{~Hz}, 1 \mathrm{H}), 2.75(\mathrm{dd}, J=13.5,7.0 \mathrm{~Hz}$, $1 \mathrm{H}) ;{ }^{13} \mathrm{C}$ NMR (125 MHz, $\mathrm{d}_{6}$-DMSO): $\delta$ 167.8, 155.8, 139.9, 135.5, 130.6, 129.5, 126.8, 118.7 , 118.6, 56.9, 37.4; $\mathrm{mp} 170-173{ }^{\circ} \mathrm{C} .[\alpha]_{\mathrm{D}}^{23}=-17^{\circ}(\mathrm{c}=0.61$, in DMSO); HRMS (ESI-TOF): calculated for $\mathrm{C}_{14} \mathrm{H}_{14} \mathrm{~N}_{3} \mathrm{O}_{2}[\mathrm{M}+\mathrm{H}]^{+}$256.1081, found 256.1086. 


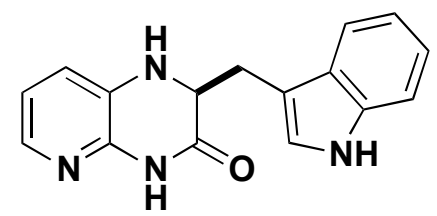

\section{(S)-2-((1H-indol-3-yl)methyl)-1,2-dihydropyrido[2,3-b]pyrazin-3(4H)-one (15S)}

Yield 47\% over two steps, light yellow solid; ${ }^{1} \mathrm{H}$ NMR (500 MHz, $\mathrm{d}_{6}$-DMSO): $\delta 10.8$ (s, $1 \mathrm{H}$ ), $10.6(\mathrm{~s}, 1 \mathrm{H}), 7.50(\mathrm{~d}, J=8.0 \mathrm{~Hz}, 1 \mathrm{H}), 7.47-7.46(\mathrm{~m}, 1 \mathrm{H}), 7.30(\mathrm{~d}, J=8.0 \mathrm{~Hz}, 1 \mathrm{H}), 7.10-7.09$ (m, $1 \mathrm{H}), 7.04(\mathrm{dd}, J=7.0,7.0 \mathrm{~Hz}, 1 \mathrm{H}), 6.96-6.91(\mathrm{~m}, 2 \mathrm{H}), 6.71(\mathrm{dd}, J=7.5,5.0 \mathrm{~Hz}, 1 \mathrm{H})$, 6.05-6.04 (m, $1 \mathrm{H}), 4.15-4.12(\mathrm{~m}, 1 \mathrm{H}), 3.09(\mathrm{dd}, J=15.0,4.0 \mathrm{~Hz}, 1 \mathrm{H}), 2.98(\mathrm{dd}, J=15.0,7.5$ $\mathrm{Hz}, 1 \mathrm{H}) ;{ }^{13} \mathrm{C}$ NMR $\left(125 \mathrm{MHz}, \mathrm{d}_{6}\right.$-DMSO): $\delta 168.2,140.0,136.1,135.5,129.5,127.5,124.1$, $120.8,118.7,118.6,118.3,118.3,111.3,109.1,56.2,28.3 ; \operatorname{mp~} 184-186{ }^{\circ} \mathrm{C} ;[\alpha]_{\mathrm{D}}^{23}=+25^{\circ}(\mathrm{c}=$ 0.48, in DMSO); HRMS (ESI-TOF): calculated for $\mathrm{C}_{16} \mathrm{H}_{15} \mathrm{~N}_{4} \mathrm{O}[\mathrm{M}+\mathrm{H}]^{+} \quad 279.1240$, found 279.1246.

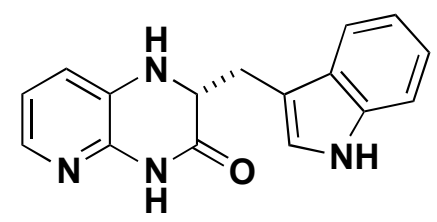

(R)-2-((1H-indol-3-yl)methyl)-1,2-dihydropyrido[2,3-b]pyrazin-3(4H)-one (15R)

Yield 52\% over two steps, light yellow solid; ${ }^{1} \mathrm{H}$ NMR (500 MHz, $\mathrm{d}_{6}$-DMSO) $\delta 10.8(\mathrm{~s}, 1 \mathrm{H})$, $10.6(\mathrm{~s}, 1 \mathrm{H}), 7.50(\mathrm{~d}, J=8.0 \mathrm{~Hz}, 1 \mathrm{H}), 7.47-7.46(\mathrm{~m}, 1 \mathrm{H}), 7.30(\mathrm{~d}, J=8.0 \mathrm{~Hz}, 1 \mathrm{H}), 7.10-7.09$ (m, $1 \mathrm{H}), 7.04(\mathrm{dd}, J=7.0,7.0 \mathrm{~Hz}, 1 \mathrm{H}), 6.96-6.91(\mathrm{~m}, 2 \mathrm{H}), 6.71(\mathrm{dd}, J=7.5,5.0 \mathrm{~Hz}, 1 \mathrm{H})$, 6.05-6.04 (m, $1 \mathrm{H}), 4.15-4.12(\mathrm{~m}, 1 \mathrm{H}), 3.09(\mathrm{dd}, J=15.0,4.0 \mathrm{~Hz}, 1 \mathrm{H}), 2.98(\mathrm{dd}, J=15.0,7.5$ $\mathrm{Hz}, 1 \mathrm{H}) ;{ }^{13} \mathrm{C}-\mathrm{NMR}\left(125 \mathrm{MHz}, \mathrm{d}_{6}\right.$-DMSO): $\delta 168.2,140.0,136.1,135.5,129.5,127.5,124.1$, $120.8,118.7,118.6,118.3,118.3,111.3,109.1,56.2,28.3 ; \mathrm{mp} 182-185^{\circ} \mathrm{C} .[\alpha]_{\mathrm{D}}^{23}=-23^{\circ}(\mathrm{c}=$ 
0.47, in DMSO); HRMS (ESI-TOF): calculated for $\mathrm{C}_{16} \mathrm{H}_{15} \mathrm{~N}_{4} \mathrm{O}[\mathrm{M}+\mathrm{H}]^{+} \quad 279.1240$, found 279.1244 .

\section{Chemical modification on the initial hit to probe SAR}<smiles>C[C@@H]1CNc2ncccc2N1</smiles>

\section{(R)-2-methyl-1,2,3,4-tetrahydropyrido[2,3-b]pyrazine $(8 R)$}

To a stirred solution of compound $1 \mathbf{R}(120 \mathrm{mg}, 0.735 \mathrm{mmol})$ in THF $(1.5 \mathrm{~mL})$ was added $\mathrm{BH}_{3} \cdot \mathrm{THF}$ solution $(1 \mathrm{M}, 3.67 \mathrm{~mL})$ dropwise. The reaction was warmed up to $65{ }^{\circ} \mathrm{C}$ and monitored by TLC until the disappearance of starting materials. The reaction was then cooled to $0{ }^{\circ} \mathrm{C}$ and quenched with $5 \mathrm{~mL} \mathrm{MeOH}$ until no more gas evolution. The solvent was removed in vacuo. The crude oil was coevaporated with $\mathrm{MeOH}(3 \times 10 \mathrm{~mL})$ to remove any excess of $\mathrm{B}(\mathrm{OMe})_{3}$. To the crude mixture $10 \mathrm{~mL}$ of $\mathrm{MeOH}$ was added followed by $20 \mathrm{~mL}$ of $\mathrm{Na} / \mathrm{K}$ tartrate $(10 \%)$ and the reaction mixture was heated at $80{ }^{\circ} \mathrm{C}$ for 10 hours. The $\mathrm{MeOH}$ was removed in vacuo and the crude was diluted with $\mathrm{NaHCO}_{3}$ solution (saturated), extracted with EA (3 times). The extract was washed with brine, dried with sodium sulfate, concentrated in vacuo and purified by column chromatography (MeOH/DCM) to afford $\mathbf{8 R}$ as yellow solid (40 mg, 36\% yield); ${ }^{1} \mathrm{H}$ $\operatorname{NMR}\left(500 \mathrm{MHz}, \mathrm{CDCl}_{3}\right): \delta 7.47(\mathrm{~d}, J=5.0 \mathrm{~Hz}, 1 \mathrm{H}), 6.63(\mathrm{~d}, J=7.5 \mathrm{~Hz}, 1 \mathrm{H}), 6.46(\mathrm{dd}, J=7.5$, $5.0 \mathrm{~Hz}, 1 \mathrm{H}), 4.87$ (bs, $1 \mathrm{H}), 3.52-3.49(\mathrm{~m}, 1 \mathrm{H}), 3.43$ (dd, $J=11.0,2.5 \mathrm{~Hz}, 1 \mathrm{H}), 3.17(\mathrm{dd}, J=$ $10.5,8.5 \mathrm{~Hz}, 1 \mathrm{H}), 1.20(\mathrm{~d}, J=6.5 \mathrm{~Hz}, 3 \mathrm{H}) ;{ }^{13} \mathrm{C} \mathrm{NMR}\left(125 \mathrm{MHz}, \mathrm{CDCl}_{3}\right): \delta 146.8,136.6$, 128.6, 118.4, 113.6, 47.4, 44.9, 19.5. m.p. $93-95{ }^{\circ} \mathrm{C} ;[\alpha]_{\mathrm{D}}^{23}=-69^{\circ}(\mathrm{c}=0.07$, in DMSO); HRMS (ESI-TOF): calculated for $\mathrm{C}_{8} \mathrm{H}_{12} \mathrm{~N}_{3} \mathrm{O}[\mathrm{M}+\mathrm{H}]^{+}$150.1026, found 150.1032 . 
<smiles>C[C@H]1Nc2cccnc2N(C)C1=O</smiles>

\section{(R)-2,4-dimethyl-1,2-dihydropyrido[2,3-b]pyrazin-3(4H)-one (9R)}

Yield $79 \%$ via the Ullmann reaction/cyclodehydration route starting with 3-bromo- $N$ methylpyridin-2-amine, brown solid; ${ }^{1} \mathrm{H}$ NMR (500 MHz, $\mathrm{d}_{6}$-DMSO): $\delta$ 7.75-7.74 (m, $1 \mathrm{H}$ ), 7.08-7.06 (m, $1 \mathrm{H}), 6.92-6.89(\mathrm{~m}, 1 \mathrm{H}), 6.38(\mathrm{~s}, 1 \mathrm{H}), 3.98$ (q, J=6.5 Hz, $1 \mathrm{H}), 3.35$ (s, $3 \mathrm{H})$, $1.32(\mathrm{~d}, J=7.0 \mathrm{~Hz}, 3 \mathrm{H}) ;{ }^{13} \mathrm{C}$ NMR $\left(125 \mathrm{MHz}, \mathrm{d}_{6}-\mathrm{DMSO}\right): \delta 168.4,141.1,136.0,131.5,119.2$, 118.8, 50.9, 27.0, 17.9. m.p. $64-67^{\circ} \mathrm{C} ;[\alpha]_{\mathrm{D}}^{23}=-101^{\circ}(\mathrm{c}=0.57$, in DMSO); HRMS (ESI-TOF): calculated for $\mathrm{C}_{9} \mathrm{H}_{12} \mathrm{~N}_{3} \mathrm{O}[\mathrm{M}+\mathrm{H}]^{+}$178.0975, found 178.0979 .<smiles>Cc1nc2cccnc2[nH]c1=O</smiles>

\section{2-Methylpyrido[2,3-b]pyrazin-3(4H)-one (10)}

To a solution of $8 \%$ aqueous sodium hydroxide $(1.0 \mathrm{~mL})$ was added compound $\mathbf{1 R}(48 \mathrm{mg}, 0.3$ mmol) followed by a solution of $30 \mathrm{wt} \%$ hydrogen peroxide in water $(0.6 \mathrm{~mL})$. The reaction mixture was slowly heated to $80{ }^{\circ} \mathrm{C}$ and maintained at this temperature for $4 \mathrm{~h}$. The heating source was removed, and acetic acid $(0.5 \mathrm{~mL})$ was added dropwise. The suspension was stirred overnight at room temperature and the precipitated solid was collected by filtration to afford the desired product 10 as white solid (20 mg, 42\% yield); ${ }^{1} \mathrm{H}$ NMR (500 MHz, $\mathrm{d}_{6}$-DMSO): $\delta 12.7$ (bs, $1 \mathrm{H}), 8.46(\mathrm{~s}, 1 \mathrm{H}), 8.10(\mathrm{~d}, J=7.5 \mathrm{~Hz}, 1 \mathrm{H}), 7.33-7.31(\mathrm{~m}, 1 \mathrm{H}), 2.40(\mathrm{~s}, 3 \mathrm{H}) ;{ }^{13} \mathrm{C}$ NMR (125 MHz, $\mathrm{d}_{6}$-DMSO): $\delta 160.7,156.2,149.1,144.0,135.8,126.8,119.5,20.5$; HRMS (ESITOF): calculated for $\mathrm{C}_{8} \mathrm{H}_{8} \mathrm{~N}_{3} \mathrm{O}[\mathrm{M}+\mathrm{H}]^{+}$162.0667, found 162.0666 . 


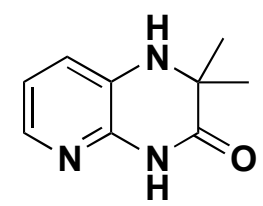

\section{2,2-Dimethyl-1,2-dihydropyrido[2,3-b]pyrazin-3(4H)-one (11)}

Yield $50 \%$ via the Ullmann reaction/cyclodehydration route starting with 2-amino-2methylpropanoic acid, light yellow solid; ${ }^{1} \mathrm{H}$ NMR (500 MHz, $\mathrm{d}_{6}$-DMSO): $\delta 10.6$ (s, $\left.1 \mathrm{H}\right), 7.62-$ $7.61(\mathrm{~m}, 1 \mathrm{H})$, 7.01-7.00 (m, $1 \mathrm{H}), 6.85-6.82(\mathrm{~m}, 1 \mathrm{H}), 6.25(\mathrm{~s}, 1 \mathrm{H}), 1.27(\mathrm{~s}, 6 \mathrm{H}) ;{ }^{13} \mathrm{C}-\mathrm{NMR}$ (125 MHz, $\mathrm{d}_{6}$-DMSO): $\delta 171.7,141.2,136.9,130.2,120.0,119.3,55.2,26.0$; HRMS (ESITOF): calculated for $\mathrm{C}_{9} \mathrm{H}_{12} \mathrm{~N}_{3} \mathrm{O}[\mathrm{M}+\mathrm{H}]^{+}$178.0975, found 178.0981.

\section{Biochemical and biophysical assays}

Biochemical assay:

The assay kit was acquired from Promega V9103, ADP-Glo ${ }^{\mathrm{TM}}$.

First, $4 \mu \mathrm{L} /$ well of CABPE, $2 \mu \mathrm{L}$ of ATP (Promega V9103 component, in AB, $53 \mu \mathrm{M}$ for GSK3 $\beta$ ), and $4 \mu \mathrm{L}$ of ligand (2.5 mM in AB with 5\% DMSO for single point inhibition), DMSO (5\% in $\mathrm{AB}$ ), or positive control (GW8510, Sigma G7791, $20 \mu \mathrm{M}$ in AB with 5\% DMSO) was dispensed into respective wells of a 384-well plate (Corning 3572) to start the reaction. The reaction was incubated at room temperature for 5 minutes. Second, $10 \mu \mathrm{L} /$ well of ADP-Glo reagent was added to terminate the reaction. The plate was incubated at room temperature for 40 minutes. Lastly, $20 \mu \mathrm{L} /$ well of kinase detection reagent was added. After 30 minutes incubation at room temperature, luminescence was read on an Envision (PerkinElmer) plate reader. Buffer conditions: $\mathrm{AB}: 25 \mathrm{mM}$ tris(hydroxymethyl)aminomethane (Tris), $10 \mathrm{mM} \mathrm{MgCl}$, $\mathrm{pH}$ adjusted to 
7.5. CABPE (in AB): $12.5 \mathrm{mM}$ dithiothreitol (Sigma 43816), $0.25 \mathrm{mg} / \mathrm{mL}$ bovine serum albumin (Sigma A4503), $0.5 \mathrm{U} / \mathrm{mL}$ heparin (Baxter NDC 0641-2440-41), GSM (GSK3 substrate peptide, Millipore 12-533, $18 \mu \mathrm{M}$ for GSK3 $\beta$ ), $18 \mathrm{nM}$ GSK3 $\beta$ (XTAL Biostructures, $46.7 \mathrm{kDa}, 1.2$ $\mathrm{mg} / \mathrm{mL}$ ). For dose-response curve determination, $15 \mathrm{R}$ and $\mathbf{1 5 S}$ were tested at final concentrations of $1.25 \mathrm{mM}, 1 \mathrm{mM}, 0.75 \mathrm{mM}, 0.5 \mathrm{mM}, 0.25 \mathrm{mM}, 125 \mu \mathrm{M}, 62.5 \mu \mathrm{M}, 31.2 \mu \mathrm{M}, 15.6 \mu \mathrm{M}, 7.81$ $\mu \mathrm{M}, 3.91 \mu \mathrm{M}, 1.95 \mu \mathrm{M}, 0.98 \mu \mathrm{M}$, and $0.488 \mu \mathrm{M}$.

First, $4 \mu \mathrm{L} /$ well of Enzyme Mix in KBA, $2 \mu \mathrm{L}$ of ATP (Promega V9103 component, in KBA, $191 \mu \mathrm{M}$ ), and $4 \mu \mathrm{L}$ of ligand (at various concentrations in KBA with 5\% DMSO), DMSO (5\% in KBA), or positive control (GW8510, Sigma G7791, $20 \mu \mathrm{M}$ in KBA with 5\% DMSO) was dispensed into respective wells of a 384-well plate (Corning 3572) to start the reaction. The reaction was incubated at room temperature for 20 minutes. Second, $10 \mu \mathrm{L} /$ well of ADP-Glo reagent was added to terminate the reaction. The plate was incubated at room temperature for 40 minutes. Lastly, $20 \mu \mathrm{L} /$ well of kinase detection reagent was added. After 30 minutes incubation at room temperature, luminescence was read on an Envision (PerkinElmer) plate reader. Buffer conditions: KBA: $50 \mathrm{mM}$ 2-[4-(2-hydroxyethyl)piperazin-1-yl]ethanesulfonic acid (HEPES), 10 $\mathrm{mM} \mathrm{MgCl}$, $\mathrm{pH}$ adjusted to 7.5, $1 \mathrm{mM}$ EGTA, 0.01\% BRIJ-35. Enzyme Mix (in KBA): CDK5 (38 nM, complex with $\mathrm{P} 25,83.8 \mathrm{kDa}$, from K Kosik lab), Histone $\mathrm{H} 1$ peptide (100 $\mu \mathrm{M}$, PKTPKKAKKL, ordered from AnaSpec, MW = 1138.5). For dose-response curve determination (Figure S2), 15R and $\mathbf{1 5 S}$ were tested at final concentrations of $1.25 \mathrm{mM}, 1 \mathrm{mM}, 0.75 \mathrm{mM}, 0.5$ $\mathrm{mM}, 0.25 \mathrm{mM}, 125 \mu \mathrm{M}, 62.5 \mu \mathrm{M}, 31.2 \mu \mathrm{M}, 15.6 \mu \mathrm{M}, 7.81 \mu \mathrm{M}, 3.91 \mu \mathrm{M}, 1.95 \mu \mathrm{M}, 0.98 \mu \mathrm{M}$, and $0.488 \mu \mathrm{M}$. 
Differential scanning fluorimetry assay:

DSF experiments were performed on a 480 Roche Lightcycler in a 384 well format. The fragments were tested at $2.5 \mathrm{mM}$ with $2.5 \%(\mathrm{v} / \mathrm{v})$ DMSO. The optimal condition was determined to be $0.12 \mathrm{mg} / \mathrm{mL}$ GSK3 $\beta$ and 1:400 dilution of Sypro Orange from the original stock. The assay was performed with final volume of $5 \mu \mathrm{L}$ using a buffer solution containing 50 mM HEPES (pH 7.5) and $150 \mathrm{mM} \mathrm{NaCl}$. Fragments that gave a thermal shift greater $0.5{ }^{\circ} \mathrm{C}$ were considered hits. Compounds 10, 13, 14, and 15 were tested at $1.25 \mathrm{mM}$ due to solubility limits.

\section{Isothermal titration calorimetry:}

ITC experiments were performed on an ITC $_{200}$ instruments from Microcal Inc. (GE Healthcare) at $25{ }^{\circ} \mathrm{C}$. The ITC cell was loaded with GSK3 $\beta$ in concentrations of $50-85 \mu \mathrm{M}$ with $2.5-5 \%$ (v/v) DMSO solution. Ligands were tested at $0.5-2 \mathrm{mM}$ concentrations. Typically, 18 injections of $2.4 \mu \mathrm{L}$ ligand were performed over a period of $30 \mathrm{~min}$ with stirring at $1000 \mathrm{rpm}$.

\section{E. Crystallization, structure determination and refinement}

Diffraction quality crystals of GSK3 $\beta$ were obtained by sitting drop vapor diffusion at $17^{\circ} \mathrm{C}$ by mixing $2 \mu \mathrm{L}$ of protein solution with $1 \mu \mathrm{L}$ reservoir solution (20\% PEG-3350, $0.2 \mathrm{M}$ sodium formate) and allowing for equilibration over $400 \mu \mathrm{L}$ of reservoir. Crystals began to appear after one week; two weeks later, crystals were incubated overnight with the addition of $0.3 \mu \mathrm{L} \mathbf{1 R}$ (200 mM in DMSO). Crystals were flash-cooled in liquid nitrogen with $10 \%$ glycerol as cryoprotectant and diffraction data were collected at the X29 beam line of the National Synchrotron 
Light Source (Brookhaven National Laboratory, Upton, NY), using a ADSC Q315 CCD detector and $1.0750 \AA$ radiation.

Crystals of GSK3 $\beta / \mathbf{1 5 R}$ complex were obtained by co-crystallization. The protein:inhibitor solution was prepared by mixing $40 \mu \mathrm{L} 6 \mathrm{mg} / \mathrm{ml}$ recombinant GSK3$\beta$ with $1 \mu \mathrm{L} 40 \mathrm{mM} \mathbf{1 5 R}$ in DMSO and $0.8 \mu \mathrm{L} 100 \mathrm{mM}$ TCEP. Diffraction quality crystals of the GSK3 $\beta: \mathbf{1 5 R}$ complex were obtained by sitting drop vapor diffusion at $17^{\circ} \mathrm{C}$ by mixing $1 \mu \mathrm{L}$ of the protein:inhibitor solution with $1 \mu \mathrm{L}$ reservoir solution (20\% PEG-3350, 0.2 M dipotassium phosphate) and allowing for equilibration over $400 \mu \mathrm{L}$ of reservoir. After 4 days, the crystals were harvested. Cryo-protection and data collection for the crystals of GSK3 $\beta: \mathbf{1 5 R}$ complex were the same as for the GSK3 $\beta / \mathbf{1 R}$ complex.

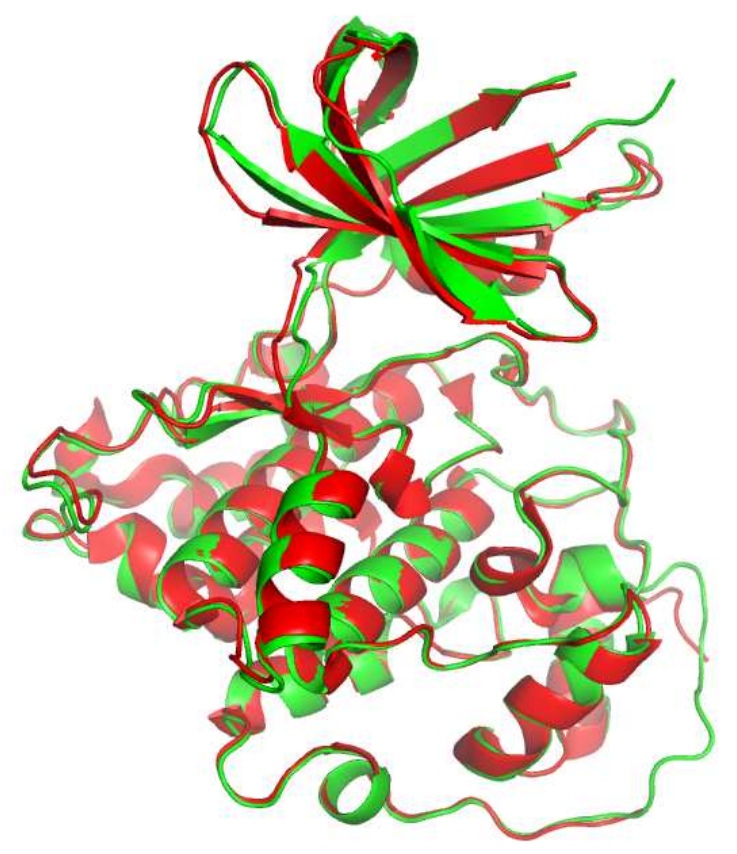


Figure S3. Structural comparisons of GSK3 $\beta$. The structure of GSK3 $\beta$ in complex with $\mathbf{1 5 R}$ (green ribbon) is superimposed with that of GSK3 $\beta$ in complex with a 1,3,4-oxadiazole derivative (red ribbon in $\mathrm{A}$ ).

All diffraction data were integrated and scaled with the HKL2000 software package. ${ }^{7}$ An initial structure of the GSK3 $\beta$ structure was determined by molecular replacement with the program PHASER, ${ }^{8}$ using the GSK3 $\beta$ structure (PDB ID: $1 \mathrm{GNG}$ ) as the search model. Ligand models were generated with the PRODRG2 server. ${ }^{9}$ The final model was built with $\mathrm{COOT}^{10}$ and refined with REFMAC5. ${ }^{11}$ Data collection and refinement statistics are presented in Table S1. Atomic coordinates and structure factors of the GSK3 $3 / \mathbf{1 5 R}$ and GSK3 $\beta / \mathbf{1 R}$ complexes have been deposited to the PDB and are available under the accession code 4J1R and 4J71.

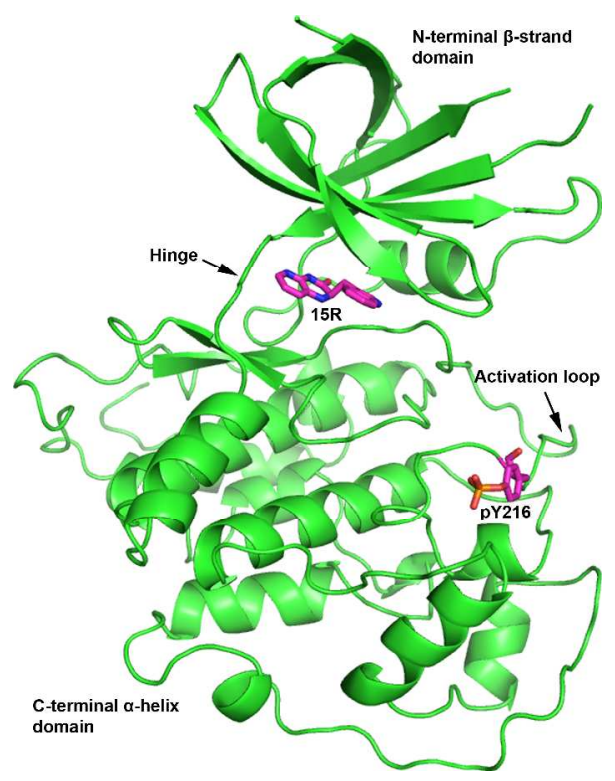

Figure S4. Structure of GSK3 $\beta$ in complex with $\mathbf{1 5 R}$. The structure of GSK3 $\beta$ is shown in green ribbons. Compound 15R and phosphotyrosine Y216, are displayed in stick representation. 
The structure of GSK3 $\beta$ in complex with $\mathbf{1 5 R}$ was determined in a new crystal form. The overall structure is highly similar to previously reported GSK3 $\beta$ structures, with the most closely related structure being GSK3 $\beta$ in complex with a 1,3,4-oxadiazole derivative (PDB ID 3F7Z; RMSD value of $0.62 \AA$ over $334 \mathrm{C} \alpha$ residues (Figure S3)).

Table S1. Statistics for data collection and refinement

Data collection

\begin{tabular}{|l|l|l|}
\hline & GSK3 $\beta / \mathbf{1 5 R}$ & GSK3 $\beta / \mathbf{1 R}$ \\
\hline Space group & $\mathrm{P} 1$ & $\mathrm{P} 1$ \\
\hline Unit-cell lengths $(\AA)$ & $\begin{array}{l}\mathrm{a}=67.5 \mathrm{~b}=67.4 \mathrm{c}=116.4 \\
\alpha=90.13 \quad \beta=90.08 \gamma=81.11\end{array}$ & $\begin{array}{l}\mathrm{a}=63.38 \mathrm{~b}=67.12 \mathrm{c}=67.44 \\
\alpha=99.80 \quad \beta=102.94 \gamma=89.94\end{array}$ \\
\hline Resolution range $(\AA)$ & $2.70-50.00$ & $2.30-50.00$ \\
\hline Highest resolution sell $(\AA)$ & $2.70-2.75$ & $2.30-2.34$ \\
\hline Unique reflections $(\mathrm{N})$ & 54028 & 45704 \\
\hline Redundancy & 2.1 & 2.2 \\
\hline Completeness $(\%)^{1}$ & 98.0 & 97.6 \\
\hline$R_{\text {merge }}{ }^{1}, 2$ & 0.097 & 0.051 \\
\hline$<I /$ sigmaI $>^{1}$ & 8.67 & 16.18 \\
\hline
\end{tabular}

Refinement

\begin{tabular}{|l|l|l|}
\hline Resolution range $(\AA)$ & $2.70-50.0$ & $2.30-42.49$ \\
\hline$R_{\text {work }}{ }^{1,3}$ & $17.6 \%$ & $22.2 \%$ \\
\hline$R_{\text {free }}{ }^{1}$ & $19.2 \%$ & $24.1 \%$ \\
\hline Average B-factors $(\AA 2)$ & 39.29 & 54.64 \\
\hline R.m.s. bonds $(\AA)$ & 0.006 & 0.004 \\
\hline R.m.s. angles $\left(^{\circ}\right)$ & 0.881 & 0.853 \\
\hline
\end{tabular}

1. Values in parenthesis correspond to the highest resolution bin. 
2. $R_{\text {merge }}=\Sigma_{\mathrm{hkl}} \Sigma_{\mathrm{i}}\left|\mathrm{I}_{\mathrm{i}}(\mathrm{hkl})-\overline{\mathrm{I}(\mathrm{hkl})}\right| / \Sigma_{\mathrm{hkl}} \Sigma_{\mathrm{i}} \mathrm{I}_{\mathrm{i}}(\mathrm{hkl})$

3. $R_{\text {work }}=\sum\left|F_{\mathrm{c}}-F_{\mathrm{o}}\right| / \sum F_{\mathrm{o}}$.

\section{Reference}

1. Hung, A. W., et al. Route to three-dimensional fragments using diversity-oriented synthesis. Proc. Natl. Acad. Sci. USA 108, 6799-6804 (2011).

2. Zhong, C., Wang, Y., Hung, A.W., Schreiber, S. L. \& Young, D. W. Diastereoselective control of intramolecular aza-Michael reactions using achiral catalysts. Org. Lett. 13, 5556-5559 (2011).

3. Sauer, W. H. B. \& Schwarz, M. K. Molecular shape diversity of combinatorial libraries: A prerequisite for broad bioactivity. J. Chem. Inf. Comput. Sci. 43, 987-1003, (2003).

4. Sauer, W. H. B. \& Schwarz, M. K. Size doesn't matter: Scaffold diversity, shape diversity and biological activity of combinatorial libraries. Chimia 57, 276-283, (2003).

5. Chen, J. J. et al. Discovery of dihydroquinoxalinone acetamides containing bicyclic amines as potent Bradykinin B1 receptor antagonists. Bioorg. Med. Chem. Lett. 18, 4477$4481(2008)$.

6. Tanimori, S., Kashiwagi, H., Nishimura, T. \& Kirihata, M. A General and Practical Access to Chiral Quinoxalinones with Low Copper-Catalyst Loading. Adv. Synth. Catal. 352, 2531-2537 (2010).

7. Otwinowski, Z. \& Minor, W. Processing of X-ray diffraction data collected in oscillation mode. Method. Enzymol. 276, 307-326 (1997).

8. Storoni, L. C., McCoy, A. J. \& Read, R. J. Likelihood-enhanced fast rotation functions. Acta. Crystallogr. D 60, 432-438 (2004). 
9. Schuttelkopf, A. W. \& van Aalten, D. M. F. PRODRG: a tool for high-throughput crystallography of protein-ligand complexes. Acta. Crystallogr. D 60, 1355-1363 (2004).

10. Emsley, P. \& Cowtan, K. Coot: model-building tools for molecular graphics. Acta. Crystallogr. D 60, 2126-2132 (2004).

11. Murshudov, G. N., Vagin, A. A. \& Dodson, E. J. Refinement of macromolecular structures by the maximum-likelihood method. Acta. Crystallogr. D 53, 240-255 (1997). 